\title{
Operational Readiness Simulator: Optimizing Operational Availability Using a Virtual Environment
}

\author{
Shaun Horning, ${ }^{1}$ Philip Leung,, Andy Fitzgerald, ${ }^{2}$ and Nezih Mrad ${ }^{3}$ \\ ${ }^{1}$ Controls and Simulation Department, GasTOPS Ltd., Ottawa, ON, Canada K1J 9J3 \\ ${ }^{2}$ ACF Associates, Bath, ON, Canada KOH $1 G 0$ \\ ${ }^{3}$ Defence R\&D Canada, Department of National Defence, Ottawa, ON, Canada K1A OK2
}

Correspondence should be addressed to Nezih Mrad, nezih.mrad@drdc-rddc.gc.ca

Received 11 March 2012; Accepted 14 August 2012

Academic Editor: Peter Foote

Copyright (C) 2012 Shaun Horning et al. This is an open access article distributed under the Creative Commons Attribution License, which permits unrestricted use, distribution, and reproduction in any medium, provided the original work is properly cited.

The maintenance and logistics systems that support aircraft fleets are complex and often very integrated. The complexity of these systems makes it difficult to assess the impact of events that affect operational capability, to identify the need for resources that can affect aircraft availability, or to assess the impact and potential benefits of the system and procedural changes. This problem is further complicated by the adoption of condition-based maintenance approaches resulting in dynamic maintenance planning as maintenance tasks are condition directed instead of scheduled or usage based. A proof of concept prototype for an aircraft operational readiness simulator (OR-SIM) has been developed for the Canadian Forces CH-146 Griffon helicopter. The simulator provides a synthetic environment to forecast and assess the ability of a fleet, squadron, or aircraft to achieve desired flying rates and the capability of the sustainment systems to respond to the resultant demands. The prototype was used to assess several typical scenarios including adjustment of preventative maintenance schedules including impact of condition-based maintenance, variation of the annual flying rate, and investigation of deployment options. This paper provides an overview of the OR-SIM concept, prototype model, and sample investigations and a discussion of the benefits of such an operational readiness simulator.

\section{Background}

Operational availability, capability, or readiness, also known as aircraft mission capable rates, is used to describe the availability of aircraft to fly their assigned missions. Fleet operation and sustainment systems for Air Force weapon systems are highly complex and multilayered and involve stakeholders from different commands within the military, the public service, and industry. There exist three primary elements through which operational capability is generated. The first is the weapon system maintenance plan. The second is the capability and capacity of the maintenance organizations (e.g., Air Force and support contractors) to produce available aircraft through the execution of the maintenance plan. The third is the aircraft operational concept through which the Air Force uses and "consumes" the available aircraft. As shown in Figure 1, the interaction or "fit" of these three elements determines the operational capability of a given fleet. From the perspective of an operational capability model, the interaction of these elements across different armed forces weapon systems combine to determine the overall operational capability of the armed forces of a nation.

Improving the fit, and thereby increasing the capability, involves understanding the characteristics of each element and the interactions between them and then taking steps to align these elements to maximize such operational capability. Leaders and mangers at all levels make decisions that have the potential to either improve or deteriorate the existing fit of these three elements and therefore affect operational capability yet there is no readily available tool through which this impact can be assessed. The result is that potential impacts on operational capability cannot be reliably determined prior to making decisions on these changes.

Furthermore initiatives such as condition-based maintenance $(\mathrm{CBM})$ that may improve operational capability cannot be supported by any objective evidence linking the initiative to the benefit. Central to the CBM concept is the idea that maintenance planning and scheduling become 


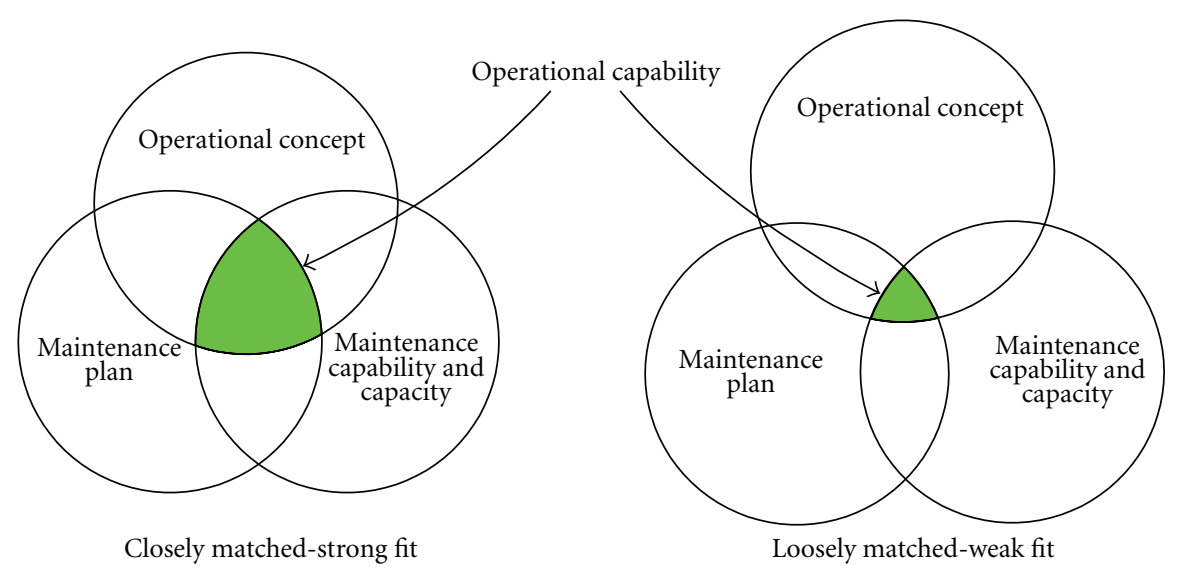

FIGURE 1: Interactions that affect operational capability.

condition directed instead of static or usage based. CBM holds the potential to significantly improve the operational not only availability and ownership costs of aircraft fleets but also increases the complexity and dynamics of fleet sustainment systems. Since with CBM, maintenance actions are driven by need, rather than on a routine or repetitive basis, the coordination of maintenance resources becomes much more dynamic as available resources are deployed to meet the emerging maintenance needs.

Therefore, there exists a need for a means to forecast and assess the ability of a fleet, squadron, or aircraft to achieve a desired operational availability and the capability of the sustainment systems to respond to the resultant demands. In addition, there is a requirement for a scenario planning and assessment capability for operations and maintenance planners, which can be used for quantitative assessments of changes to operations and sustainment plans and to provide a dynamic schedule forecasting capability to assess short- and long-term resource requirements based on forecasted aircraft flying rates. Ideally, this would be implemented in a model in a synthetic (virtual) environment where knowledge of the fleet sustainment systems can be consolidated and provide a true capability to manage operational capability instead of simply managing fleets, aircraft, or contracts.

1.1. CH-146 Griffon Helicopter. The CH-146 Griffon is the Canadian military variant of the Bell 412EP, a multiuse utility helicopter powered by two Pratt and Whitney PT6T gas turbine engines. The Griffon is a multiuse platform equipped to provide aerial support reconnaissance, search, rescue, and mobility tasks. The fleet of CH-146 Griffon Utility Tactical Transport Helicopters is divided into six squadrons spread out across Canada. Collectively, these squadrons operate under the command of 1 Wing Kingston. Five Tactical Helicopter Squadrons support specific Army brigades within their geographic regions. The sixth squadron is an operational training squadron.

While collectively the squadrons operate under the command of 1 Wing, the squadrons are self-contained and field deployable and as such each squadron operates relatively independently of the other squadrons. As each squadron fulfills slightly different roles, the number of aircraft assigned to each squadron and the number and duration of missions that the squadron flies vary resulting in different usage rates of the aircraft assigned to each squadron. Like all aircraft, the $\mathrm{CH}-146$ has a scheduled (i.e., preventative) maintenance plan. The $\mathrm{CH}-146$ preventative maintenance plan includes both usage and calendar-based inspections of key aircraft components such as airframe, engine, and gearbox. Since aircraft usage tends to vary between squadrons, the resulting preventative maintenance burden is also different.

In addition to the preventative maintenance, the squadrons must also contend with component failures, troubleshooting of issues on the $\mathrm{AC}$, and reconfiguration of the $\mathrm{AC}$ for different missions. These tasks are collectively referred to as corrective maintenance and together the preventative and corrective maintenance result in aircraft downtime (i.e., unavailable to fly missions).

The maintenance resources such as the number of repair bays, paint booths, and qualified maintenance personnel also differ between the squadrons. In each squadron, the senior squadron maintenance personnel must optimize the use of the squadron's limited maintenance resources to maintain a level of availability that best meets the demands for operational aircraft. For example, the maintenance planner must assign personnel to specific maintenance tasks to ensure that both short-term and long-term maintenance actions are completed. The squadron personnel must also attempt to assign aircraft to missions such that the upcoming scheduled maintenance for the aircraft is staggered so as to not overload the maintenance capacity of the squadron.

The squadron to squadron differences in number of aircraft, quality of aircraft, aircraft usage rates, and the number and availability of resources represents a difficult modelling challenge and necessitates the need for a data-driven model that can be configured based on the input data. The data input structures must be generic enough to handle the squadron to squadron variations.

1.2. Discrete Event Simulation (DES). The basis for discrete event simulation is a "transaction-flow world view" where a system is visualized as consisting of discrete units of traffic (or "entities") that move ("flow") from point to point in the system while competing with each other for scarce 
resources. DES is ideally suited to model a variety of systems ranging from manufacturing, supply chain, customer service, communications, and defence. The state of a model in DES changes at only discrete but possibly random sets of simulated time points. During these time points, one or more entities may move. The general structure of DES software is described in detail in textbooks and papers such as [1]. DES is ideally suited for simulating aircraft operations and support systems. The aircraft would be the entities and different points in the system would represent different processes such as maintenance, the flying of missions, and preand postflight checks. To perform an analysis using DES, one or more replications are required. A replication is the execution (running) of the model from start to completion. Multiple replications use the same model but with a different set of (generated) random numbers and so produce different statistical results. The results across multiple replications can then be analyzed to determine averages.

MathWorks provides a toolbox for its Simulink simulation environment called SimEvents [2] for the development of event-based simulations. Since Simulink is a time marching simulation environment, it is also possible to develop hybrid (continuous and event-based) simulations making it ideally suited for development of component degradation models. The use of SimEvents also leverages the data import/ export capabilities of MATLAB/Simulink to access either databases or Microsoft Excel workbooks. Aircraft components modelled in SimEvents retained all of their attributes (data) when combined and/or separated with other components. MATLAB/Simulink/SimEvents combine a powerful programming language and modelling toolset with a DES capability making it ideal for use as the modelling environment.

\section{Operational Readiness Simulator Model}

2.1. Model Overview. The operational readiness simulator (OR-SIM) is a DES model of the fleet operations and maintenance activities. The model simulates flight operations of a squadron and the resulting preventative and corrective maintenance of individual aircraft. The fault characteristics of specific components on specific aircraft are simulated creating a unique simulation of each aircraft. In addition, component degradation models allow for the assessment of condition-based maintenance burdens.

The OR-SIM model is a "closed" simulation where all the input data is preloaded at model initialization and no other (user) inputs are required while the simulation is executing. The model inputs, consisting of configuration, operation, and maintenance data, are stored in data templates that are read in on initialization and the data is then propagated throughout the model to populate the various component models. Examples of model input data include squadron and aircraft specific operations and maintenance data such as squadron nominal flying rates and aircraft component flight hours. Monte Carlo simulation techniques are then used to address the stochastic nature of this data. In addition, scenario specific time scheduled events such as the start of a fleet-wide modification can be used to modify the base data set. The model data is described in more detail in a subsequent section.

As shown in Figure 2, the model structure is divided into three main components: Aircraft Initialization, Flight Line, and Maintenance. These submodels are briefly described in the following paragraphs.

In the Aircraft Initialization model, AC subcomponent (e.g., airframes, engines, etc.) entities are created and populated with initial usage, condition indicator, scheduled maintenance, and failure data. As the entities are created, they are combined to form $\mathrm{AC}$ entities according to the configuration data by matching unique identifiers (e.g., engine serial numbers and airframe tail numbers) and the completed AC entities are sent to the Flight Line model.

The Flight Line model consists of models of the mission planning, preflight checks, mission flying, and postflight checks. The mission planning model selects suitable AC to fly as many of the day's scheduled missions as possible. The selection of $\mathrm{AC}$ in the mission planning model simulates the techniques used by the Air Force to optimize availability by assigning $\mathrm{AC}$ to missions so as to maximize use prior to time consuming calendar backstopped inspections (referred to as "stagger"). The pre- and postflight check models check the component failure flags to determine if a component failure has been detected and processes the AC based on the failure effect code. The preflight check model also includes a model of the power assurance check (PAC) that is used to update the interturbine temperature (ITT) margin condition indicator values stored for each engine on the AC.

After the preflight check, the AC entity proceeds to the fly sortie model which consists of three processes: determining in-flight failures, updating component usage, and degradation levels, and holding the $\mathrm{AC}$ to simulate the mission time. As part flying the mission, the model simulates component degradation which then results in changes to the condition indicators (e.g., ITT Margin) that are used to trigger condition-based maintenance tasks. Statistical representations of degradation rates determined as a function of mission type and duration and key environmental conditions (e.g., desert versus marine operations) are used to degrade performance as part of each mission. These relationships can be determined through analysis of historical data, experimental study, and detailed performance simulations. Once the $\mathrm{AC}$ returns from the mission, it proceeds to the postflight check model.

The Maintenance model includes a maintenance and resource planning model that simulates a maintenance manager and assigns resources to aircraft in maintenance. This model is based on techniques used by squadron maintenance managers to optimize availability by ensuring that long duration maintenance actions such as the annual inspection are continuously worked on. Each maintenance manager can have a unique approach to assigning resources to specific tasks. The model can be adjusted to evaluate different approaches to the assignment of resources to tasks. Once the resources are assigned, the aircraft and resources are processed in the maintenance work model. In the maintenance work model, the fraction of time that the maintenance personnel is working on the $\mathrm{AC}$ is simulated as 


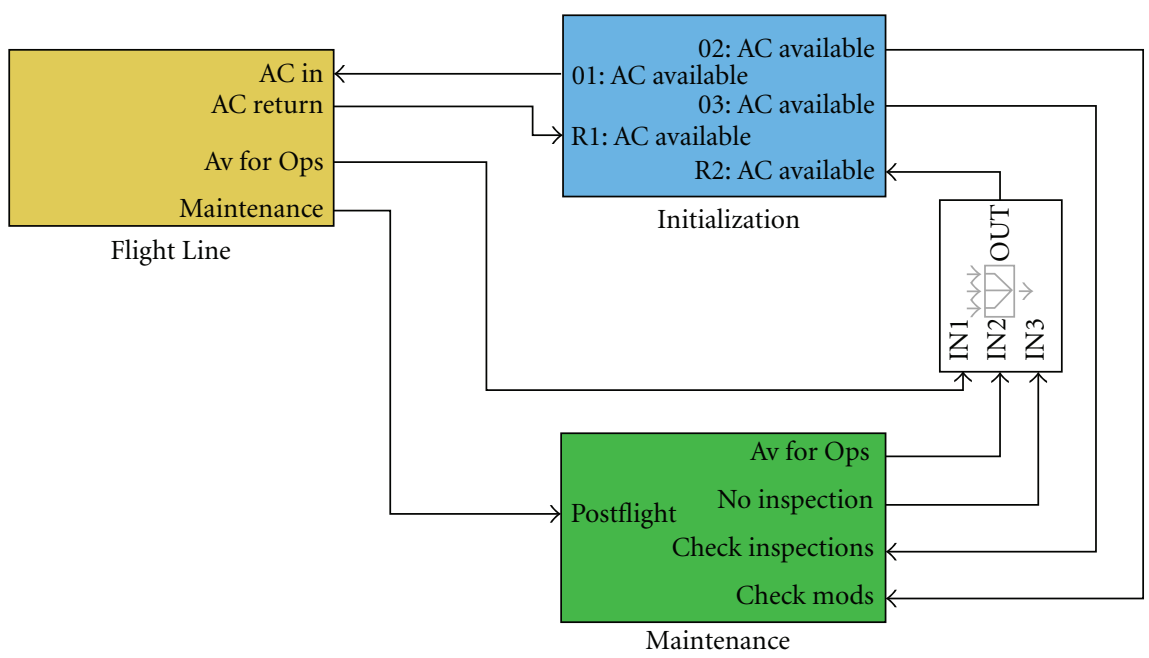

FIgURE 2: OR-SIM SimEvents model.

a contiguous period of time and interruptions are simulated by reducing the time the resource is available during the shift. For example, while maintenance personnel may work an eight hour work shift, due to other responsibilities (e.g., training, base defence, etc.) they may only be available to work on maintenance tasks $50 \%$ of the time. When all of the maintenance actions are completed, the maintenance records are closed and the maintenance flags are reset and the AC is returned to the flight line.

During run time, model parameters are continuously monitored and stored in output variables along with a time stamp so that time histories of parameters can be produced. When a test (replication) is completed, all of the model data is saved to an output results files. Results files from replication sets are then postprocessed to determine outputs suitable for direct comparison to data normally captured by the Air Force (e.g., aircraft flying hours, downtime, etc.) and can be viewed for specific actions.

2.2. Model Input Data. The OR-SIM model input data consists of three types of data: configuration, operation, and maintenance data. Configuration data is primarily used in model initialization to create and populate the AC entities. The input operational data is used to determine when AC fly and how long they fly to simulate the missions that were flown during the study period. Maintenance input data includes component failure rates, scheduled maintenance plans, and the time required to complete maintenance tasks.

The current version of the OR-SIM simulates only 4 major subassemblies (components or entities) of the $\mathrm{CH}-146$ : airframe, engine, gearbox, and rotor. These were selected primarily based on the preventative maintenance plan for the $\mathrm{CH}-146$ which is divided into airframe, engine, and gearbox inspections. To accurately model the fleet, the simulated AC entities must reflect the same configuration and usage as the fleet at the start of the study period. Each component is created at model initialization, populated with usage data such as current flying hours and condition indicator values, and assembled into AC according to the configuration data. To properly model aircraft maintenance, previous maintenance dates and component failure data are also assigned to each assembled AC entity. The configuration data is used to baseline the simulated fleet to a configuration and level of usage at some point in time. Records from Health and usage monitoring systems (HUMS) installed on the AC and maintenance records for performance or power assurance checks (PAC) can be used to obtain initial values for relevant condition indicators such as ITT Margin.

Since the AC have been in use and have undergone periodic maintenance, the preventative maintenance schedule for each AC is initialized to the date and flying hours of the last scheduled maintenance. The model can then calculate the aircraft flying hours (AFH) or calendar date of the next scheduled maintenance. The component failure rates used to trigger corrective maintenance are similarly initialized.

The operational data is a function of the operational concept or profile. This data is used to determine the number of missions and the durations of the missions to simulate the AC usage. Different mission types can be simulated and distributions are used to add variation to the flights and durations. A usage factor is used to define the ratio of AFH (engine-on-time) to the actual time that the $\mathrm{AC}$ is executing the mission. A repeating schedule of a single day, week, month, year, and so forth can be used.

The OR-SIM maintenance data consists of component failure data, scheduled maintenance plan, maintenance completion data, and squadron resource levels.

Component failure data is used to trigger corrective maintenance activities. The failure data consists of failure rates, when the failure was detected and the effect that the failure had on the mission (e.g., no effect, delay, abort, etc.). Ideally, a mean flight hours to failure and standard deviation would be calculated for each corrective maintenance type (Work Unit Code or WUC) for each AC. However, the historical failure rates were generally low so that an AC specific average and standard deviation for each WUC for all AC could not be calculated. Therefore, a method based on dividing and categorizing the fleet based on failure rate was used to 
obtain the average and standard deviation component failure rates. A discrete distribution was used to model the effect of the failure for each WUC.

For each component, the scheduled maintenance (inspection) can be triggered based on usage and/or calendar time. For each inspection, the usage and calendar time between inspections are required. In addition a usage and calendar time window are used to define a range of usage or time when the inspection would be done if the aircraft was already in maintenance. A hierarchy of concurrent scheduled maintenance is used to trigger inspections that are performed as part of other scheduled maintenance (e.g., lower usage interval inspections are performed when higher usage interval inspections are triggered).

The maintenance completion data is used by the model to determine the number of maintenance person hours (MPHR) that are required to complete each of the simulated maintenance actions. For each maintenance action, the average, standard deviation, minimum, and maximum MPHR are input into the model. A minimum calendar time value is also included to simulate delays in the parts supply network that was not modelled. For each maintenance action, minimum and maximum numbers of workers are also defined. The maintenance completion data for the ORSIM was obtained from the historical maintenance records stored in the Air Force maintenance repository database.

The input data is stored in Microsoft Excel spreadsheets that are loaded on model initialization. The use of spreadsheets provides a means to input data in an organized and readily understandable format. The configuration and maintenance data required for the model was obtained from existing databases that track aircraft configuration and maintenance records (e.g., failure rates and maintenance action completion times). The Excel tables match airframe tail number to component (e.g., engine and gearbox) serial numbers and can be easily updated with changes in configuration. The maintenance records are preprocessed offline to calculate the required model data and saved to the Excel tables. Similarly, the preventative maintenance plan was also defined in a table in Excel that matched component inspections with usage and calendar backstops. Historical operational records of flight times by aircraft were used to create operational profiles of flights that were used by the Flight Line model to simulate the flying rate of the AC (usage hours). Squadron specific data (e.g., number of resources, available repair bays, etc.) are stored in a MATLAB data (.m) file. Different scenarios can be simulated by manipulating the input data (e.g., adding/removing inspections, increasing flying rate, etc.).

2.3. Model Output Data. The OR-SIM outputs can be calculated for an individual aircraft tail number, for a group of specific aircraft (e.g., squadron), or for the entire fleet of aircraft. Since the OR-SIM models each AC individually during the study period, almost any data regarding $\mathrm{AC}$ condition and location can be tracked during the simulation. For example, it is possible to examine an individual AC throughout the study period to determine when the AC was idle, when it was flying, and when it entered maintenance (and the cause for maintenance) and to track its usage and performance/condition indicators such as ITT Margin. For convenience the outputs are grouped in the following categories: summary, operational, and maintenance.

The summary information calculated from the OR-SIM model includes common indicators of fleet performance including the total number of calendar hours that the AC spends in preventative and corrective maintenance and the operational availability (ratio of calendar hours where the aircraft is available to fly missions to the total number of calendar hours). In many cases the output must be calculated in a given time period (e.g., flying hours may be calculated per month or per year). In these cases the outputs can be calculated for specific periods and averaged (e.g., average monthly flying rate per year). In other cases the specific output is defined only at a particular instant (e.g., Preventative Maintenance/Inspection Stagger Charts). Time outputs such as MPHR and aircraft downtime can be expressed in time (e.g., hours or days) or as a relative measure (e.g., percentage of downtime due to corrective maintenance).

In addition to the summary information, the OR-SIM can output other important data including total AFH, number of mission aborts, number of maintenance hours and the total number of hours the AC are unavailable due to corrective or preventative maintenance, maintenance personnel utilization rates, AC usage between calendar-based inspections, and the time from when a modification order is issued to when the last $\mathrm{AC}$ receives the modification. In addition, condition indicators can be monitored to determine when maintenance was triggered to assess the effectiveness of the CBM program.

These outputs are directly comparable to the metrics used in the quarterly fleet health reports and therefore the simulation results can be used to essentially forecast the fleet health reports for different scenarios. The model summary outputs can be compared to the actual fleet health reports to evaluate and support decisions, identify bottlenecks in the organization, and predict availability.

2.4. Model Validation. The OR-SIM was validated against historical data from two squadrons over a two-year period. The objective was to predict overall results consistent with average trends found in actual fleet data. In particular, it was intended for the prototype model to predict total maintenance hours and maintenance actions and total flight hours within $10 \%$ of the fleet data.

To ensure proper model initialization or "warm-up," the model was initialized with data from one year previous to the validation period. The warm-up period ensures that the modelled system has reached equilibrium representative of normal operations and any assumptions in the initial data no longer have an impact on the output of the system. The simulation was executed for 3 years starting with twenty replications of simulation. The initial seeds for the random number generators in the model (e.g., for determining flight duration, MPHR for maintenance actions, etc.) were randomized between replications. Once all of the replications were complete, the model outputs from each replication were processed to determine the average and the $90 \%$ confidence 
interval. In general the simulation results match well with the historical records and were typically within the $10 \%$ target.

For the first squadron, the simulated squadron total AFH, downtime, and operational availability for the first year were within $2 \%$ of the fleet totals. The total maintenance MPHR was within $10 \%$ of the fleet total, although the corrective maintenance results were overpredicted by $16 \%$. Since the model is based on average values based over several years of mean time between failure data, it cannot replicate the short-term differences in failure times that can occur in one year. The difference in actual and simulated corrective MPHR could be reduced by further refining the component fault data and possibly using more sophisticated probability distributions (e.g., Weibull) rather than the normal distribution used in the model. Since the purpose of the study was to develop a proof of concept model structure for an operational availability simulator, a rigorous analysis of the failure data was not performed and normal distributions were generally assumed. Further analysis of the reliability data may show that other distribution types provide a better fit and these can be easily accommodated with minor updates to the model and input data.

There was also some variation in the preventative maintenance MPHR distribution among the periodic maintenance types. These differences may be caused by assumptions in the initialization data, the use of waivers to extend intervals between periodic maintenance, which was not modelled, or variations in the recording of the maintenance data.

In the second year the airframe hours were again within $2 \%$, although the availability was overpredicted by $7 \%$. The maintenance burdens were similar to the previous year so it is possible that the drop in availability was due to a reduced maintenance capacity (i.e., maintenance personnel), or parts availability - neither of which were accounted for in the simulation input data. These influences could be further investigated, but since the results were within the validation target of $10 \%$ they were outside the scope of the prototype model.

Similar results were observed for the second squadron. The first-year model results matched the historical operational availability, downtime, total airframe hours, and total corrective MPHR within 2\% or less. However, the model overpredicted the total preventative MPHR. Detailed analysis of the historical preventative maintenance records show significantly less than the expected number of monthly airframe inspections. This finding requires further investigation. The second-year validation results were similar.

\section{Scenario Investigations}

Once the OR-SIM was validated, it was used to investigate several different scenarios. In each scenario, the effects of a change to the fleet baseline on key fleet performance parameters such as availability, yearly flying rate, maintenance hours, and aircraft downtime were investigated. Examples of scenarios included adjustment of calendar backstops on periodic maintenance, effect of changes to squadron operating profiles (increases/decreases in flying rates), effect of deployment on operational capacity, assessment of implementation methods for a modification, and extension of engine wash intervals based on power assurance check (PAC) results. The results of two of these investigations are discussed below.

3.1. Aircraft Flying Hours Variation. This scenario investigated the sensitivity of operational availability and preventative and corrective maintenance burdens to changes in annual flying rate. As the aircraft flying rate was changed it was expected that there would be a variation in aircraft availability and in the ratio of preventative and corrective maintenance. The aircraft flying rates were revised by $\pm 10 \%$ and $\pm 25 \%$ of the nominal value by adding or removing flights. A 10-year simulation of the fleet was executed and compared to the fleet baseline. For reference, a set of 20 replications was completed in approximately 19 hours on a standard single core 2010 era desktop computer. A sample of results from the simulation is shown in Figure 3.

In general, increases in flying rate resulted in decreases in operational availability but the squadron was able to still achieve the planned flying rates up to $125 \%$ of the nominal illustrating that it is possible for the squadron to support sustained increased operations with the current maintenance capacity. It is interesting to note that a $10 \%$ reduction in flying rate resulted in negligible increases in operational availability. The amounts of corrective and usage-based scheduled maintenance are functions of usage and hence flying rates. However, calendar-based scheduled maintenance is relatively constant. For the $\mathrm{CH}-146$ the annual inspection represents approximately $50 \%$ of the total scheduled maintenance MPHR and is unaffected by usage or flying rate. The model shows that a minor reduction in flying rate does not reduce the remaining required scheduled maintenance enough to increase the availability.

This is an example of using the OR-SIM to forecast the effect of annual flying rate on availability and maintenance burden. It can be used to assess the capacity of the squadron to support higher flying rates and can be used to forecast the expected preventative and corrective maintenance hours that will be required to support the new flying rates. The model can also be used to identify aspects of the maintenance plan (e.g., calendar backstops on preventative maintenance) that can lead to lower availability even with reduced flying rates.

3.2. Engine Wash. In this scenario, the OR-SIM model was used to investigate the effect of increasing the engine wash interval as well as to simulate the impact of on-condition washes. The CH-146 maintenance plan includes an oncondition compressor wash triggered by data collected by the onboard HUMS. The PAC is an aircrew function performed with the aircraft light on skids on a daily basis (i.e., first flight of the day) to verify the engine performance. Data collected by the HUMS for each PAC is used to calculate the engine ITT margin which is used to evaluate the engine performance. The engine wash also has an operating hours limit and analysis has shown that the degradation rate between washes was negligible and so the majority of the engine washes were completed due to this schedule requirement rather than on-condition. 


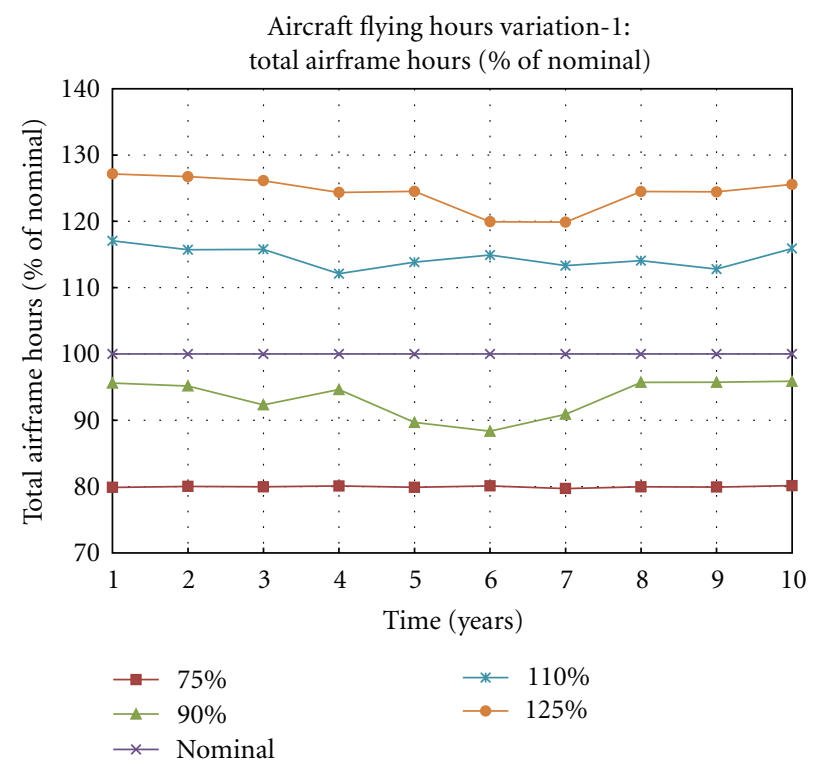

(a)

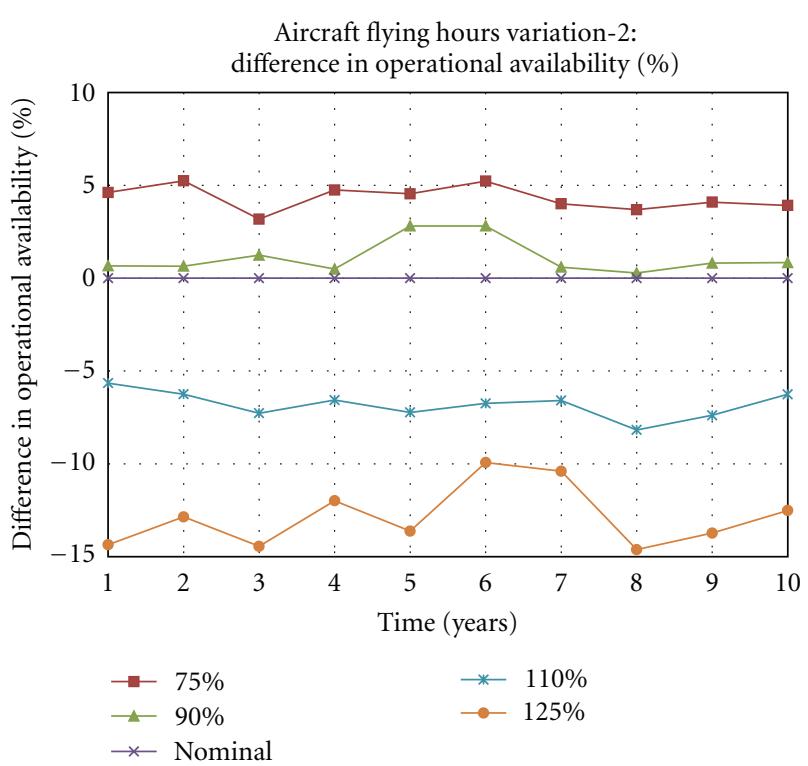

(b)

FIgURE 3: Aircraft flying hours and operational availability for variations in yearly flying rate.

TABLE 1: Extension of engine wash interval results.

\begin{tabular}{|c|c|c|c|c|}
\hline \multirow{4}{*}{$\begin{array}{l}\text { Parameter } \\
\text { (change relative to default } \\
\text { degradation rate and schedule) }\end{array}$} & \multicolumn{3}{|c|}{ 10-Year average } & \\
\hline & \multirow{2}{*}{\multicolumn{2}{|c|}{$\begin{array}{c}\text { Default ITT margin } \\
\text { Degradation rate }\end{array}$}} & \multirow{2}{*}{\multicolumn{2}{|c|}{$\begin{array}{c}\text { Increased ITT margin } \\
\text { Degradation rate }\end{array}$}} \\
\hline & & & & \\
\hline & Default schedule & Extended schedule & Default schedule & Extended schedule \\
\hline Case & $\mathrm{A}$ & B & $\mathrm{C}$ & $\mathrm{D}$ \\
\hline Operational availability & - & $1 \%$ & $-1 \%$ & $0 \%$ \\
\hline Down time & - & $-3 \%$ & $3 \%$ & $0 \%$ \\
\hline Total airframe hours & - & $0 \%$ & $0 \%$ & $0 \%$ \\
\hline Total maintenance MPHR & - & $-1 \%$ & $0 \%$ & $-1 \%$ \\
\hline Total washes & - & $-34 \%$ & $15 \%$ & $-15 \%$ \\
\hline Scheduled washes (\% total) & $100 \%$ & $100 \%$ & $74 \%$ & $57 \%$ \\
\hline On-condition washes ( $\%$ total $)$ & $0 \%$ & $0 \%$ & $26 \%$ & $43 \%$ \\
\hline
\end{tabular}

As part of this scenario investigation, the airframe maintenance schedules were revised to extend the operating hours limit. Since it can be expected that there would be an increase in the ITT margin degradation rate due to the extension of the operating hours limit, the model was also modified to investigate the influence of ITT margin degradation rate. It is expected that the change in degradation rate would increase the frequency of on-condition compressor washes. A 10-year simulation of the fleet was executed. In this scenario, it was assumed that the level of effort to complete the engine wash was constant between scenarios and there would be no change in fault rate of aircraft components. The results for the two compressor wash intervals for no degradation and with component degradation are summarized in Table 1.

Comparing Case B to Case A, assuming no significant degradation in the ITT margin, as expected, extending the wash schedule resulted in a reduction in the number of engine washes and a slight increase in availability due to the reduced preventative maintenance burden. Also as expected, since the degradation rate remained the same, there were no on-condition washes. Case B represents the ideal case where the extension of the wash schedule had no impact on engine performance and would result in a $34 \%$ reduction in the number of washes. The likelihood of that is expected to be relatively low since it can be expected engine performance would be affected by having a dirty compressor/engine. These two cases (A and B) were run to provide a comparison for the cases where the degradation rate was changed.

For a simulation with an increased degradation rate and the default wash schedule (Case C), there were a number of on-condition washes which resulted in an increase in the total number of washes compared to the default case (Case A). This increase in the number of washes resulted in a decrease in availability but marginal change in the overall maintenance burden. 
When the wash schedule was extended with the increased degradation rate (Case D), the total number of washes was found to decrease and a higher percentage of washes were completed on-condition compared to the previous case (Case C). Compared to the default case (Case A), even with the increased degradation rate the availability was unchanged and the overall maintenance burden was slightly decreased. Compared to Case A, the $15 \%$ less engine washes would also reduce the materiel costs for the wash fluid.

Based on these results, one could conclude that it can be expected that the extension of the compressor wash interval would yield a reduction in compressor washes of up to $34 \%$ (Case B) but likely would be closer to $15 \%$ because it is expected that the engine performance would be affected by the increased interval. To validate these results, the schedule would have to be increased and the ITT margins would have to be monitored to determine the actual degradation rate due to the extended wash interval. Once the degradation rate has been determined, this analysis can be repeated to determine the expected reduction in the total number of washes or the ratio of on-condition to scheduled washes. This scenario highlights the potential of an OR-SIM model to predict the effects of proposed changes to a CBM plan on aircraft availability and maintenance hours.

\section{Summary}

A CH-146 operations model was developed as a proof of concept prototype for an Operational Readiness Simulator. The model was validated against historical flight and maintenance data records for two squadrons over a two-year period. Overall, the model provided a reasonable match to the historical data. The simulated squadron total AFH, downtime, and operational availability were typically within $2 \%$ of the fleet totals. Predicted total maintenance labour hours were within approximately $10 \%$ with some differences in the distribution of preventative and corrective activities. Total preventative maintenance MPHR matched well indicating that the total preventative workload is being modelled but the historical records showed variability in the individual inspection records. The validated model successfully demonstrated that it could be used to investigate representative scenarios.

This project has developed several technological advances that incorporate a number of unique attributes and capabilities that are not readily found in previous discrete event simulations applied to the domain of fleet operations and sustainment. In particular, the incorporation and use of condition data obtained from embedded health and usage monitoring systems and the integration of process models spanning operations, logistics, and maintenance are expected to greatly enhance the fidelity and scope of the simulated operations and sustainment processes.

This work has demonstrated that an integrated model can simulate operations, scheduled, corrective, and condition-based maintenance in a synthetic environment. The fundamental framework including the procedures and techniques for modelling the operations, maintenance-and in particular the maintenance decision-making processes that was developed for this project can be used to model other fleets or organizations. The OR-SIM model serves as a basis for models to provide the fleet managers a synthetic environment to quantify the cost-benefit tradeoffs during the decision-making processes required to manage the operations and maintenance for a fleet of vehicles or other group of complex machinery. This further allows the fleet managers the ability to assess changes to the maintenance including the assessment of impacts of condition-based maintenance on mission capability and operating costs.

Another use for an OR-SIM-type model is as a training tool to illustrate the operations of the fleet and provide candidate maintenance managers a synthetic environment to apply their understanding of the maintenance processes and receive immediate feedback on their performance.

\section{Future Plans}

This OR-SIM project addressed the base simulation component of the OR-SIM. There are additional simulation components as well as other components of the OR-SIM platform yet to be investigated including a user interface (for use by fleet managers and candidate maintenance managers who may not be familiar with the simulation platform). Currently the OR-SIM model is very technical and requires a user that is knowledgeable and comfortable in the modelling language to develop scenarios, execute the model, and analyze the results. The user interface component is a key component in ensuring that the target users and fleet managers are provided a means to execute the model with only a very minimal knowledge of the details and technology of the base simulation. This phase of the development would require input from the target users to ensure that the interface meets their needs and expectations. In particular the model results processing and results display will need further refinement. Web-based model hosting is also a possible component to ensure availability of the OR-SIM to users.

Additional simulation components not developed in this project include the component models primarily related to logistics (e.g., sparing/inventory management models and organization transfer models). These are models that can be developed to expand the scope of the OR-SIM model.

As previously mentioned, other possible areas of further development and analysis include refining the data collection and data reduction processes (e.g., distributions).

\section{References}

[1] T. J. Schriber and D. T. Brunner, "Inside discrete-event simulation software: how it works and why it matters," in Proceedings of the Winter Simulation Conference, pp. 14-22, December 1997.

[2] SimEvents for MATLAB/Simulink by The MathWorks, 2011, http://www.mathworks.com/products/simevents/?s_cid=HP_ FP_SL_SimEvents Retrieved: April 2011. 

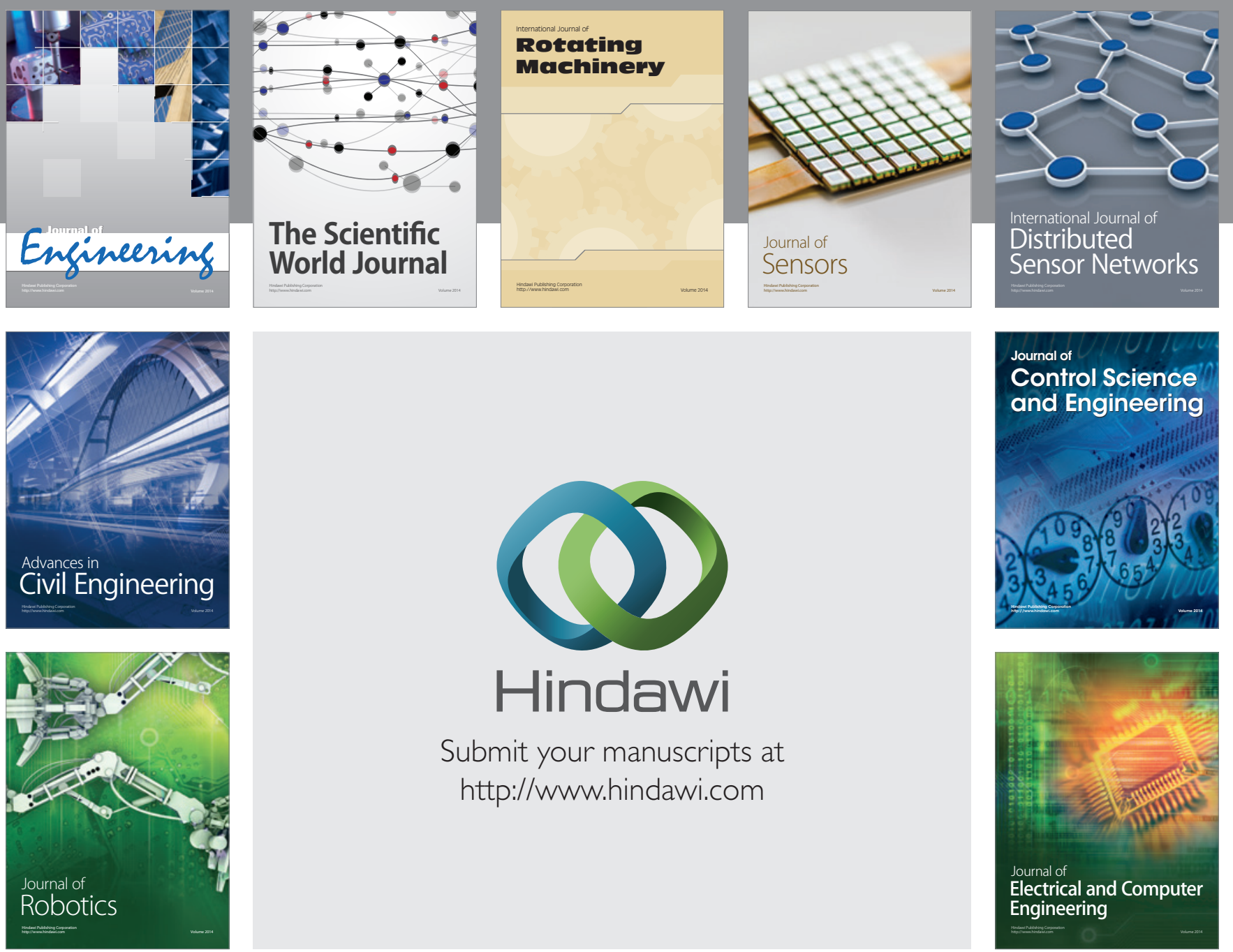

Submit your manuscripts at

http://www.hindawi.com
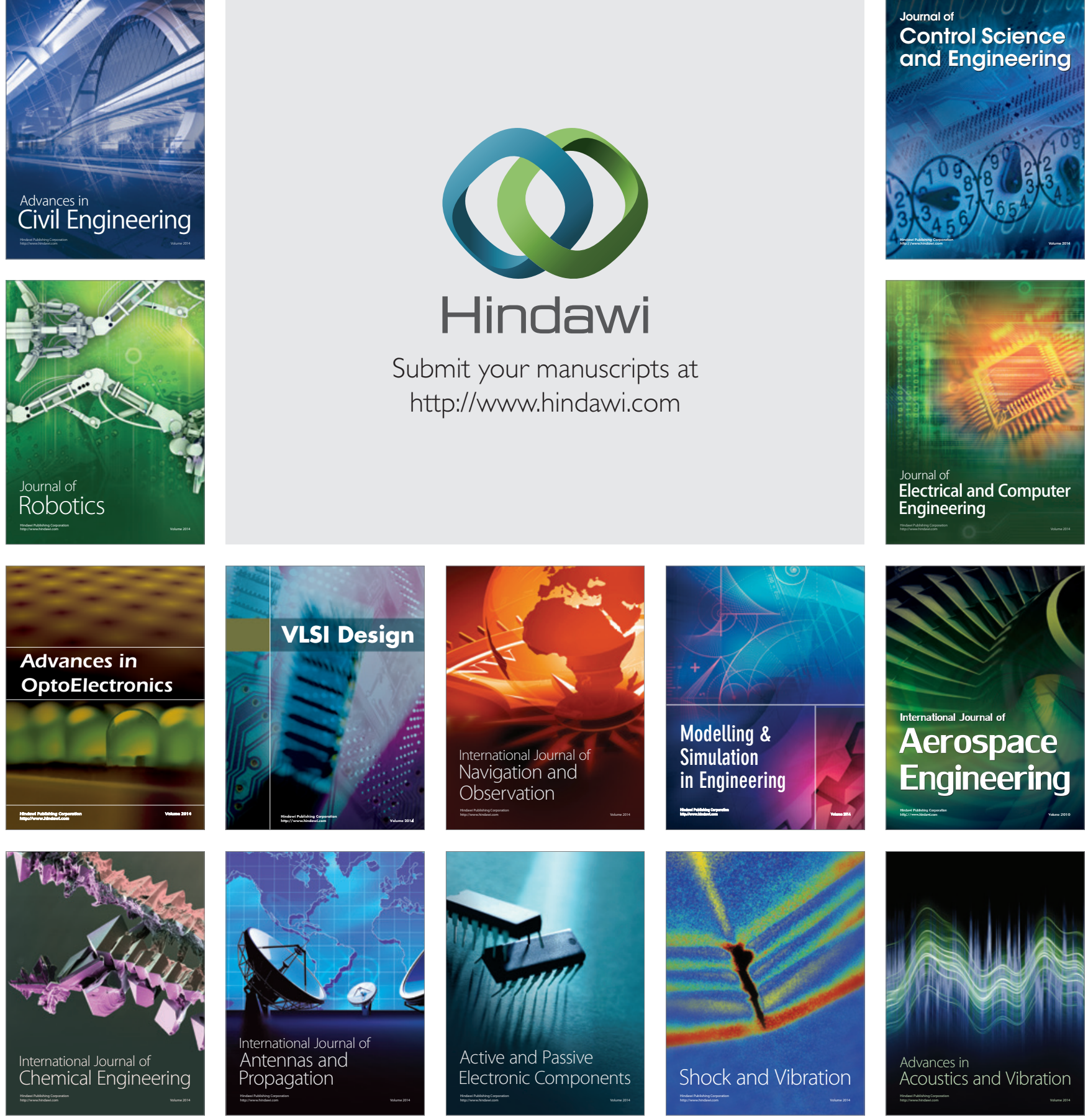